\title{
Neglect Benevolence in Human-Swarm Interaction with Communication Latency*
}

\author{
Phillip Walker ${ }^{1}$, Steven Nunnally ${ }^{1}$, Mike Lewis ${ }^{1}$, Andreas Kolling ${ }^{2}$, Nilanjan \\ Chakraborty $^{2}$, and Katia Sycara ${ }^{2}$ \\ 1 University of Pittsburgh, Pittsburgh, PA 15213, USA. \\ 2 Carnegie Mellon University, Pittsburgh, PA 15213, USA.
}

\begin{abstract}
In practical applications of robot swarms with bio-inspired behaviors, a human operator will need to exert control over the swarm to fulfill the mission objectives. In many operational settings, human operators are remotely located and the communication environment is harsh. Hence, there exists some latency in information (or control command) transfer between the human and the swarm. In this paper, we conduct experiments of human-swarm interaction to investigate the effects of communication latency on the performance of a human-swarm system in a swarm foraging task. We develop and investigate the concept of neglect benevolence, where a human operator allows the swarm to evolve on its own and stabilize before giving new commands. Our experimental results indicate that operators exploited neglect benevolence in different ways to develop successful strategies in the foraging task. Furthermore, we show experimentally that the use of a predictive display can help mitigate the adverse effects of communication latency.
\end{abstract}

\section{INTRODUCTION}

Swarm robotic systems are composed of simple individual units and generate collective behavior that is robust to failure of individual robots [1,2]. However, for practical use of such systems in large and complex human-supervised missions, key problems that arise in human-swarm interaction need to be understood and solved. In application scenarios, the human operator may be remotely located and there may be communication constraints due to the hardware limitations of the robots (e.g., communication radios of limited power) and the environmental properties (e.g. underwater environments). This will lead to delay in the communication of information between the swarm and the human. The delay in communication results in the human neither knowing perfectly the current state of the swarm nor the effect of her action on the robots. The extant literature on Human-Swarm Interaction (HSI) [3-10] has not studied the performance and behavior of human operators in the presence of delayed information transmission between the swarm and the human and vice versa. Therefore, in this paper,

* This research has been sponsored in part by AFOSR FA955008-10356 and ONR Grant N0001409-10680. 
we create an experimental scenario to study the effects of latency and error on human performance in controlling swarm robotic systems. We also study the use of predictive displays to mitigate the effect of latency.

In our experimental foraging scenario, a human operator guides a swarm to find unknown targets in a given area. The robots have a single behavior, namely flocking, and the operator applies inputs (a) to give a desired direction of flocking to the robots and (b) to enforce cohesiveness among the robots (by activating constraints for attracting neighbors that are far away and repelling neighbors that are very close). In our experiment, each subject performs the mission under three conditions, namely, (a) without any latency (control condition), (b) with equal latency in the human to swarm and swarm to human communication channel (c) the same latency as (b) but with a predictive display. In all conditions, each robot has some error in transforming the orientation heading to its own reference frame (due to localization errors), which is modeled as a Gaussian distribution. Our experimental results indicate that, as expected, there is a degradation of performance due to latency. However, when using the predictive display, the performance of the operators can be as good as it was in the absence of delay (control condition). We also found that the users exhibited different strategies for effectively controlling the swarm.

The human operator needs to influence the swarm without adversely disturbing the swarm (such as breaking it into many small connected components). The effect of an operator command is dependent on swarm state, which gradually evolves to a steady state after a command has been issued. To capture the idea that humans may need to observe the evolution of the swarm state before acting, we investigate a novel concept called neglect benevolence, whereby neglecting the swarm to allow for stabilization before issuing new commands may be beneficial to overall mission performance. An analogous but different concept called neglect tolerance $[11,12]$ is used in human robot interaction. For independently operating multiple robots, neglect tolerance is defined as the time a human can neglect a robot without degradation in system performance. For neglect tolerance, it is assumed that the performance of an individual robot degrades with time and hence the attention of the operator needs to be scheduled so that the time between servicing robots is minimized $[13,14]$. In contrast, neglect benevolence captures the concept that it may be beneficial to leave the swarm alone for a certain length of time after issuing an instruction to allow the behavior to stabilize (since the swarm state may not degrade monotonically with time). Our results show evidence of neglect benevolence.

\section{TASK DESCRIPTION}

Our study investigates the ability of a human operator to effectively influence a swarm operating under algorithms that require time to exhibit emergent behaviors. In particular, we investigated (1) the effect of communication latency in human-swarm performance, (2) the effect of predictive displays, and (3) the existence of neglect benevolence as a new notion in HSI. We created a foraging 
task that requires users to direct a swarm around an open environment using instructions to change swarm heading and flocking constraints. We also use this study to look at the effect of communication latency on this ability.

\subsection{The Environment}

The overall task of the experiment is to guide the swarm around an open, 100x100 meter environment to find targets of different colors. We use three different environments divided into six regions, with each region containing one of three target frequencies: low (0-4 targets), medium (5-9 targets), or high (10+). The target distribution is different across the search missions that each participant solves, but each environment contains 1 high, 2 low, and 3 medium frequency regions. There are 40 targets in total in each of the three environments, and each participant receives a worksheet indicating the target frequency of each region.

We use Stage v. 3.2.2 [15] to simulate the environment, the targets, and a swarm of 40 differential drive P2AT robots. Robot controllers are implemented using the Robot Operating System (ROS) [16]. Each robot is equipped with a color sensor with a range of 4 meters to detect the colored targets. We also simulate an additional sensor that allows the robots to sense the location of a neighbor within 4 meters. This allows each robot to estimate the direction of motion of its neighbors from repeated observations of their location.

The graphical user interface is also implemented in ROS. This interface displays the known targets and positions of the robots; however, it does not display the region boundaries. During the trial, each robot transmits its position and observations from its color sensor to the user interface. A target is considered found only if six or more robots detect it simultaneously, at which point the target is shown on the map and a counter on the side is incremented.

\subsection{Human Influence}

Users can influence the swarm with three commands: stop, heading, and applyconstraints. The heading command broadcasts a global heading to the swarm. To simulate a localization error, every robot interprets the global heading with respect to a local coordinate frame to compute its goal heading. The orientation of this local coordinate frame differs from the true orientation of the robot by an error sampled from the Gaussian distribution $\mathcal{N}\left(0, \frac{4 \pi}{9}\right)$. Upon receiving the command, the robots turn toward their respective goal heading and begin moving (Fig. 1a). In order to correct for the erroneous interpretations of the global heading, each robot also executes a consensus algorithm at a frequency of 0.5 Hertz. Robots sense the direction of motion of their neighbors and update their goal heading to the average goal heading of their neighbors and themselves (Fig. 1b). Finally, the user can issue an apply-constraints command, which applies biologically-inspired flocking constraints similar to those in [1], [2], and [10]. These constraints force robots to repel from each other if they were closer than 1.5 meters, and, if none were this close, to attract to neighbors further than 3 
meters (fig. 1c). Otherwise, the robots execute the consensus algorithm described previously.

a)

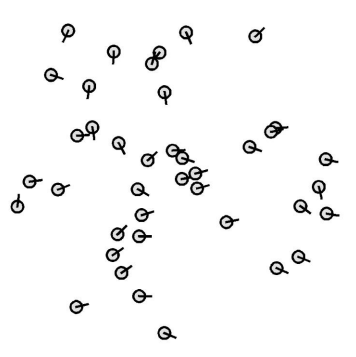

b)

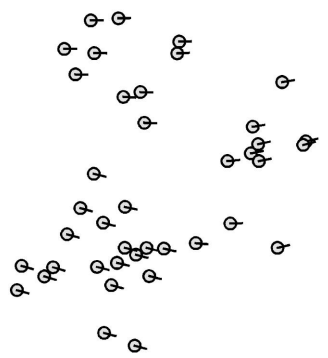

c)

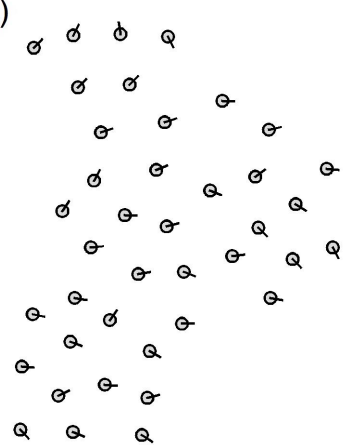

Fig. 1. The swarm in each of the three possible states: when the user first issues a heading command (a), after the consensus algorithm has stabilized (b), and after the user has just issued the constraints command (c). Before a heading command is issued, the robots headings (indicated by the solid line coming out of the circles) can be in different directions (a). After consensus, all the headings align in the same direction (b). When a constraints command is issued, headings may drift away from consensus temporarily as the robots adjust to the constraints.

\subsection{Experimental Design}

The experiment consists of three conditions - the control, latency, and predictive conditions. In all conditions, the operator begins with a swarm of 40 robots positioned randomly in a 10x10 meter box.

In the control condition, there is no latency in either of the human-to-swarm or swarm-to-human channels.

In the latency condition each channel-operator-to-swarm and swarm-tooperator - has a latency of 10 seconds, meaning that operator-issued commands will not reach the robots until 10 seconds after issuing, and the state of the swarm displayed in the interface for the user is 10 seconds old. Therefore, from the operator's point of view, the swarm will not begin executing the command until 20 seconds after the heading instruction is issued, as the message will take 10 seconds to reach the swarm, and the reflection of this command will take 10 more seconds to return to the operator.

In the predictive condition, the latency of 10 seconds for each channel remains present; however, the interface gives the user a prediction of where each member of the swarm will be in 20 seconds, or when the next command is received, by taking the heading and speed (which is a constant $0.5 \mathrm{~m} / \mathrm{s}$ ) of each swarm member and extrapolating the robot's position that far in the future. 
Each participant has a different environment for each of these conditions, and the order of both the conditions and the maps are randomized for each participant in order to remove any learning biases. 21 participants $(8$ men and 13 women) were recruited from the greater Pittsburgh area to participate in the study. Each participant was given a short explanation of the controls and goals of the study and a 10-minute training session to familiarize themselves with the interface, after which they completed each of the three conditions.

\section{RESULTS AND DISCUSSION}

The overall mission performance for each participant is measured in terms of the number of targets found and the coverage of the high-frequency target regions. In the control condition participants found 19.86 targets and covered $1.47 \mathrm{~m}^{2} / \mathrm{s}$ of the high-frequency target regions on average, both of which were significantly higher than in the latency condition, where participants found 16.71 targets $(p=.021)$ and covered $0.98 \mathrm{~m}^{2} / \mathrm{s}$ of the high-frequency regions $(p=.007)$. In the predictive condition, however, participants found 18.86 targets on average and covered $1.24 \mathrm{~m}^{2} / \mathrm{s}$ of the high-frequency regions, neither of which were significantly different from the control condition ( $p=.467$ and $p=.196$, respectively). These results show that the latency of 10 seconds significantly impedes performance, but that the predictive display in the predictive condition removes this impediment.

An indirect measure of an operator's ability to control the swarm is the swarm's overall connectivity. To determine the overall connectivity of the swarm at any given time, we represented the swarm as a simple graph, $G$, and used the second eigenvalue of the graph's Laplacian as the connectivity measure. Keeping the swarm connected has two benefits. First, such a swarm is less likely to break off into smaller connected components, which allows the user to meet the sixrobot threshold for sensing a target more easily. Secondly, a highly-connected swarm will reach consensus faster, as each robot will have more neighbors to average with during each consensus round.

We see that the latency condition had an average connectivity of 0.111 , which was significantly higher than in control condition, which had an average connectivity of $0.084, p<.001$. Similarly, average connectivity in the predictive condition was also significantly higher than in the control condition, with a value of $0.116, p<.001$, see Fig. 2. This points to the existence of neglect benevolence, as it demonstrates that communication latency helped enforce swarm connectivity by causing operators to wait to see the results of their heading command before deciding whether to issue a new one. As a consequence, each command has a longer duration, thus giving the swarm more time to stabilize after each command. We find this is indeed true, with users issuing significantly more commands on average in the control condition $(M=27.81)$ than in the latency condition $(M=17.76, p=.028)$, and significantly more than in the predictive condition $(M=18.86, p=.052)$. 

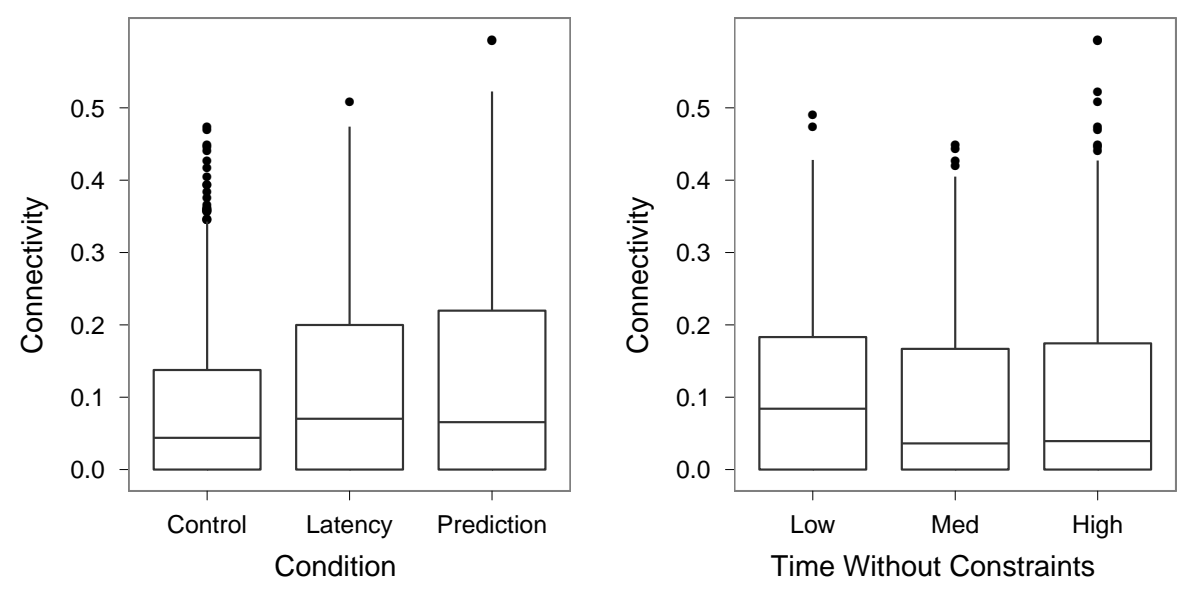

Fig. 2. These graphs display the connectivity of the swarm at the end of each heading command issued. Connectivity is significantly different across conditions (left), with the latency conditions generally having more connected swarms. The average time to constraints also impacts connectivity (right), with participants choosing to enforce constraints more often generally having better connected swarms. The boxes in each figure represent the bottom three quartiles, and the outliers are marked as black dots.

To investigate the various behaviors and strategies of the operators, we investigated the duration and timing of the heading and apply-constraints instructions. We analyzed the average time between a heading and a subsequent apply-constraints command (hereafter referred to as time to constraints), or the next heading command (duration). Because these instructions involve altering the current state of the swarm at the time they are issued, even two identical commands (i.e., two heading commands with the same heading) can lead to drastically different effects depending on the state of the swarm.

As demonstrated by the previous results, because the number of heading commands were different across condition, the duration of the commands are similarly different. The apply-constraints instruction, however, can be more flexible, and operators may decide to issue constraints at any point in time after a heading command, or issue a new heading command without activating constraints at all. To investigate the effect of the application of constraints, we clustered the data into three equal-sized groups across all missions into a high ( $100 \%$ to $78 \%$ ), medium ( $78 \%$ to $45 \%$ ), or low (45\% to $0 \%$ ) group corresponding to the number of heading commands for which constraints were later applied.

Performance in terms of targets found does not differ between these groups, but the total area swept is significantly different, with fewer constraints (low) leading to less overall area covered by at least six sensors $(p=.040)$. On the other hand, many constraints (high) lead to a larger error between the heading of the swarm and the operator's goal heading $(p=.011)$, and fewer constraints (low) have more heading instructions leading to a consensus $(74 \%, p<.001)$. 
These results suggest that operators employ different strategies to find a larger number of targets, with some operators using constraints earlier and more often, increasing coverage at the expense of higher heading errors, while others preferred the opposite.

We expected a difference in heading duration and time to constraints between the two latency conditions and the control condition, as participants must wait 20 seconds to see the results of their commands. Interestingly, however, across all instructions, only $27 \%$ in the latency condition and $30 \%$ in the predictive condition have constraints activated later than 20 seconds, neither of which were significantly different from control, meaning that, unlike with the heading commands, operators often issued the constraints prior to seeing the effect of the heading instruction on the swarm. Swarm connectivity was also significantly impacted by the application of constraints as well, with the high constraints group having significantly better connectivity than the medium or low group $(p=.043)$, see Fig. 2.

These results provide considerable support for neglect benevolence. Frequent and short commands provide an operator more control, but sacrifice swarm cohesion as reflected in the lower connectivity value and the higher number of connected components. This is largely due to the fact that new heading commands reintroduce error into the swarm members' estimated heading and require several rounds of consensus to stabilize. Activating the constraints too early and often, however, leads to higher heading errors, and thus may make the swarm more difficult for the human to control. We found that operators develop two different strategies around neglect benevolence: either stabilize the consensus and lower the heading error, or maintain swarm cohesion and improve coverage. It appears operators were able to use either method to their advantage and obtain a good performance, and that, while latency can degrade performance overall, it does not impact one strategy more than the other.

\section{CONCLUSIONS AND FUTURE WORK}

This study provides support for the idea of neglect benevolence, with the commands in the study leading to strategies with different costs and benefits depending on the state of the swarm at the time the commands were issued. Frequent heading commands provided the user more control over the direction and location of the swarm at the expense of total coverage and swarm connectivity. Due to the nature of the swarm algorithms, high position and heading accuracy and high swarm cohesion were not possible simultaneously. Therefore, participants had to decide which characteristics were more important. For the present study, both strategies achieved success; however, other tasks may be better achieved with one or the other. This will be the subject of future study.

Latency had a negative effect on the number of targets found; however, using a predictive display mitigated the negative effects. Latency also seemed to significantly impact the frequency with which an operator issues heading commands, but not apply-constraints commands. As this is the first study to investigate 
latency in human-swarm interaction, future work will address latency issues for human control of other tasks and swarm algorithms.

\section{References}

1. Reynolds, C.: Flocks, herds and schools: A distributed behavioral model. In: ACM SIGGRAPH Computer Graphics. Volume 21., ACM (1987) 25-34

2. Couzin, I., Krause, J., James, R., Ruxton, G., Franks, N.: Collective memory and spatial sorting in animal groups. Journal of theoretical biology 218(1) (2002) 1-11

3. Bashyal, S., Venayagamoorthy, G.: Human swarm interaction for radiation source search and localization. In: Swarm Intelligence Symposium, 2008. SIS 2008. IEEE, IEEE (2008) 1-8

4. Kolling, A., Nunnally, S., Lewis, M.: Towards human control of robot swarms. In: Proceedings of the 7th international conference on Human-robot interaction, ACM (2012)

5. Coppin, G., Legras, F.: Autonomy spectrum and performance perception issues in swarm supervisory control. Proceedings of the IEEE (99) (2012) 590-603

6. Cummings, M.: Human supervisory control of swarming networks. In: 2nd Annual Swarming: Autonomous Intelligent Networked Systems Conference. (2004)

7. Klarer, P.: Flocking small smart machines: An experiment in cooperative, multimachine control. Technical report, Sandia National Labs., Albuquerque, NM (United States) (1998)

8. Kira, Z., Potter, M.: Exerting human control over decentralized robot swarms. In: Autonomous Robots and Agents, 2009. ICARA 2009. 4th International Conference on, IEEE (2009) 566-571

9. Naghsh, A., Gancet, J., Tanoto, A., Roast, C.: Analysis and design of human-robot swarm interaction in firefighting. In: Robot and Human Interactive Communication, 2008. RO-MAN 2008. The 17th IEEE International Symposium on, IEEE (2008) 255-260

10. Goodrich, M., Pendleton, B., Sujit, P., Pinto, J.: Toward human interaction with bio-inspired robot teams. In: Systems, Man, and Cybernetics (SMC), 2011 IEEE International Conference on, IEEE (2011) 2859-2864

11. Xu, Y., Dai, T., Sycara, K., Lewis, M.: Service level differentiation in multi-robots control. In: Intelligent Robots and Systems (IROS), 2010 IEEE/RSJ International Conference on, IEEE (2010) 2224-2230

12. Dai, T., Sycara, K., Lewis, M.: A game theoretic queueing approach to selfassessment in human-robot interaction systems. In: Robotics and Automation (ICRA), 2011 IEEE International Conference on, IEEE (2011) 58-63

13. Mitchell, P., Cummings, M.: Management of multiple dynamic human supervisory control tasks. In: 10th International Command and Control Research and Technology Symposium. (2005)

14. Mau, S., Dolan, J.: Scheduling for humans in multirobot supervisory control. In: Intelligent Robots and Systems, 2007. IROS 2007. IEEE/RSJ International Conference on, IEEE (2007) 1637-1643

15. Gerkey, B., Vaughan, R., Howard, A.: The player/stage project: Tools for multirobot and distributed sensor systems. In: Proceedings of the 11th international conference on advanced robotics, Portugal (2003) 317-323

16. Quigley, M., Conley, K., Gerkey, B., Faust, J., Foote, T., Leibs, J., Wheeler, R., Ng, A.: Ros: an open-source robot operating system. In: ICRA Workshop on Open Source Software. Volume 3. (2009) 